
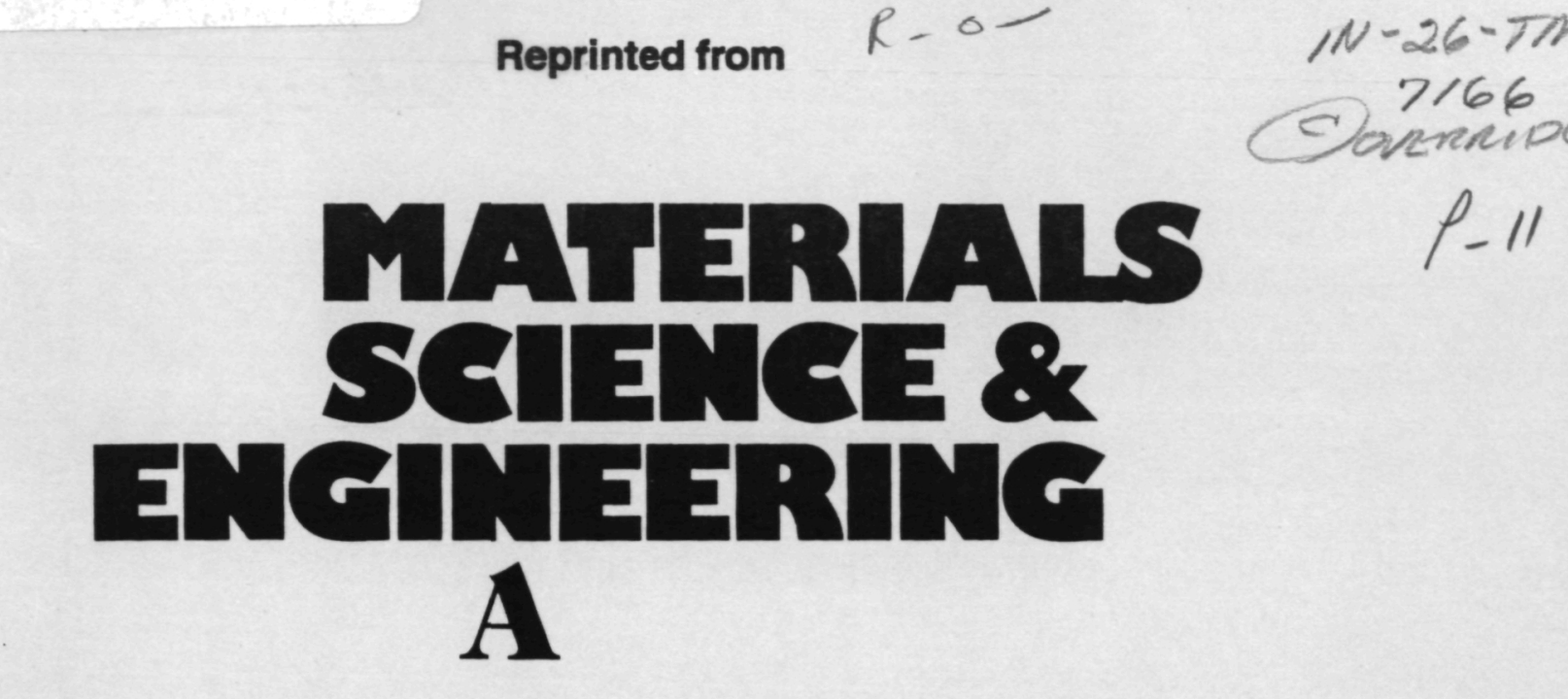

Reactive melt infiltration of silicon-molybdenum alloys into microporous carbon preforms

\author{
M. Singh ${ }^{\mathrm{a}}$, D.R. Behrendt ${ }^{\mathrm{b}}$
}

${ }^{a}$ Department of Materials Science and Engineering, Case Western Reserve University, Cleveland, OH 44106, USA

${ }^{\mathrm{b}}$ National Aeronautics and Space Administration, Lewis Research Center, Cleveland, OH 44135, USA

(NASA-TM-111193) REACTIVE MELT

INFILTRATION OF SILICON-MOLYBDENUM

ALLOYS INTO MICROPOROUS CARBON

PREFORMS (NASA. Lewis Research

Center) $11 \mathrm{P}$
N96-17149

Unclas

G3/26 0092344 


\section{MATERIALS SCIENCE AND ENGINEERING A}

The journal provides an international medium for the publication of theoretical and experimental studies and reviews of the properties and behavior of a wide range of materials, related both to their structure and to their engineering application. The varied topics comprising materials science and engineering are viewed as appropriate for publication: these include, but are not limited to, the properties and structure of crystalline and non-crystalline metals and ceramics, polymers and composite materials.

\section{Editor-in-Chief \\ Professor H. Herman \\ Regional Editors \\ M. Koiwa (Japan) \\ G. Kostorz (Switzerland)}

\section{Associate Editors (MSE A)}

R. J. Arsenault (USA)

D. Brandon (/srael)

H. K. D. H. Bhadeshia (UK)

J. G. Byrne (USA)

J. Cadek (Czech Republic)

J. B. Cohen (USA)

J. Driver (France)

J. D. Embury (Canada)

H. Fischmeister (Germany)

H. Gleiter (Germany)

C. M. Hansson (Canada)

H. Hu (USA)

Y. G. Kim (Korea)

H. Kimura (Japan)

C. Laird (USA)

M. McLean (UK)

T. Mori (Japan)

H. Oikawa (Japan)

R. C. Pohanka (USA)
L. Priester (France)
P. Rama Rao (India)
T. Sakuma (Japan)
S. Sampath (USA)
V. K. Sarin (USA)
L. L. Seigle (USA)
P. Shen (Taiwan)
S. Suresh (USA)
N. S. Stoloff (USA)
M. Taya (USA)
A. K. Vasudévan (USA)
B. Wilshire (UK)
S. Wojciechowski (Poland)
M. Yamaguchi (Japan)
T. S. Yen (China)

\section{Administrative Editor \\ Barbara Herman}

\author{
Advisory Board (MSE A and B) \\ H. Herman, Chairman (USA) \\ H. Curien (France) \\ M. E. Fine (USA) \\ A. Kelly, FRS (UK) \\ H. Mughrabi (Germany) \\ H. Rangu (Japan)
}

\section{Types of contributions}

Original research work not already published; plenary lectures and/or individual papers given at conferences; reviews of specialized topics within the scope of the journal; engineering studies; letters to the editor.

\section{Subscriptions}

Volumes $189-204$, each volume containing 2 issues, are scheduled for publication. Prices are available from the publishers upon request. Subscriptions are accepted on a prepaid basis only. Issues are sent by SAL (Surface Air Lifted) mail wherever this service is available. Airmail rates are available upon request. Please address all requests regarding orders and subscription queries to:

\section{ELSEVIER SCIENCE SA}

P.O. Box 564, 1001 Lausanne, Switzerland

Telephone: (21) 3207381

Telex: 450620 ELSA CH Telefax: (21) 3235444

Issues are sent by surface mail after air delivery to Argentina, Australia, Brazil, Canada, China, Hong Kong, India, Israel, Japan,
Malaysia, Mexico, New Zealand, Pakistan, Singapore, South Africa, South Korea, Taiwan, Thailand and the USA. Airmail rates for other countries are available on request.

For advertising rates apply to the publishers. A specimen copy will be sent on request.

US and Canadian customers may obtain information from the following.

\section{ELSEVIER SCIENCE INC.}

Attn.: Journal Information Center, 655 Avenue of the Americas New York, NY 10010, USA.

Telephone (212) 6333750 Telex: 420643 AEP UI

Telefax: (212) 6333764

\section{Abstracting and/or Indexing Services}

American Ceramic Society; Cambridge Scientific Abstracts; Chemical Abstracts; Current Contents; Engineering Index; FIZ Karlsruhe; Fluid Abstracts; Fluidex; Glass Technology Abstracts; Inspec/Physics Abstracts; Metals Abstracts; Pascal (Centre National de la Recherche Scientifique); Physikalische Berichte; Research Alert ${ }^{\mathrm{TM}}$; Science Citation Index. 


\title{
Reactive melt infiltration of silicon-molybdenum alloys into microporous carbon preforms
}

\author{
M. Singh ${ }^{\mathrm{a}}$, D.R. Behrendt ${ }^{\mathrm{b}}$ \\ ${ }^{a}$ Department of Materials Science and Engineering, Case Western Reserve University, Cleveland, OH 44106, USA \\ ${ }^{\mathrm{b}}$ National Aeronautics and Space Administration, Lewis Research Center, Cleveland, OH 44135, USA
}

Received 12 May 1994; in revised form 5 August 1994

\begin{abstract}
Investigations on the reactive melt infiltration of silicon-1.7 and 3.2 at.\% molybdenum alloys into microporous carbon preforms have been carried out by modeling, differential thermal analysis (DTA), and melt infiltration experiments. These results indicate that the pore volume fraction of the carbon perform is a very important parameter in determining the final composition of the reactionformed silicon carbide and the secondary phases. Various undesirable melt infiltration results, e.g. choking-off, specimen cracking, silicon veins, and lake formation, and their correlation with inadequate preform properties are presented. The liquid silicon-carbon reaction exotherm temperatures are influenced by the pore and carbon particle size of the preform and the compositions of infiltrants. Room temperature flexural strength and fracture toughness of materials made by the silicon-3.2 at.\% molybdenum alloy infiltration of medium pore size preforms are also discussed.
\end{abstract}

Keywords: Silicon; Molybdenum; Alloys; Carbon; Melting

\section{Introduction}

Research and development on silicon carbide based advanced ceramics and composites has attracted a great deal of attention in recent years [1-8]. The potential applications of these materials include components for advanced propulsion systems, energy conversion devices, and other high-temperature structures. For these applications, the fabrication processes for the SiC-based ceramics should yield materials which generally display high strength, high toughness, and high termal conductivity and maintain these properties under oxidizing conditions. In addition, there are other critical issues in the fabrication of silicon carbide based ceramics which are mainly related to complex shape fabricability, processing time, and temperatures. A combination of these factors leads to higher manufacturing cost of the final components. Owing to the above considerations, there is a strong need to develop processing approaches for silicon carbide based advanced ceramics which yield high strength and toughness, high thermal conductivity, good oxidation resistance, and cost effectiveness.
In terms of achieving $\mathrm{SiC}$ ceramics with desirable properties, the reaction-forming process has many advantages over other conventional silicon carbide processing techniques. This process has near-netshape and complex shape fabrication capabilities, shorter processing times, and lower processing temperatures than other conventional processes. In this technique, a microporous carbon preform is infiltrated with molten silicon or silicon-refractory metal alloys [3-5]. These molten infiltrants react with carbon to form silicon carbide or silicon carbide and refractory disilicide phases. The physical properties (pore size and volume, densities etc.) of the microporous carbon preform can be controlled to yield tailored microstructures, compositions, and properties of the final material.

Recently, for example, silicon-molybdenum alloys have been used as infiltrants instead of pure silicon [4-6] in the reactive melt infiltration processing of silicon carbide ceramics. The amount of molybdenum disilicide phase in the reaction-formed material depends on the alloy composition and the relative carbon density of the preform, which is defined as the weight of the carbon divided by the volume of the 
preform. Molybdenum disilicide as dispersed second phase provides certain advantages to the thermomechanical properties of reaction-formed silicon carbide ceramics. It has a brittle-to-ductile transition temperature around $1000{ }^{\circ} \mathrm{C}$ [9]. This phenomenon allows molybdenum disilicide particles to act as a dispersed ductile phase at high temperatures. In addition, as a dispersed phase with a thermal expansion coefficient higher than silicon carbide, it also provides toughening at lower temperatures.

In order to understand various aspects of reactive infiltration, i.e. infiltration temperature, infiltrant composition, and stability of various phases in the final material, it is very useful to have information on the phase diagrams of the molybdenum-silicon (Mo-Si) and molybdenum-silicon-carbon $(\mathrm{Mo}-\mathrm{Si}-\mathrm{C})$ systems. The phase diagram of the binary molybdenum-silicon system [10] is given in Fig. 1. The eutectic at the silicon-rich side of the diagram is at 1.7 at.\% Mo composition and the eutectic temperature is $1400{ }^{\circ} \mathrm{C}$. In order to achieve high volume fractions of molybdenum disilicide in the reaction-formed silicon carbide materials, the infiltrant composition and infiltration temperature should be chosen accordingly. Nowotny et al. [11] have reported on the high-temperature phase diagram of the $\mathrm{Mo}-\mathrm{Si}-\mathrm{C}$ system at $1600{ }^{\circ} \mathrm{C}$. In addition, there is a three-phase field containing $\mathrm{MoSi}_{2}, \mathrm{SiC}$, and $\mathrm{Si}$ indicating the stability of three phases in equilibrium.

In the reactive melt infiltration process, no external pressure is applied to the system and infiltration takes place by capillary action. The rate of infiltration depends on the wetting of the preform by the melt, which generally increases with increasing infiltration temperature. The wetting behavior of carbon and silicon carbide with silicon has been reported by a number of authors [12-15]. The contact angle of silicon on silicon carbide was reported to be $38^{\circ}$ at $1430{ }^{\circ} \mathrm{C}[13]$. Using the values of surface tension at this temperature, the work of adhesion is calculated to be $1311 \mathrm{~mJ} \mathrm{~m}^{-2}$ for the $\mathrm{Si}-\mathrm{SiC}$ system at $1430{ }^{\circ} \mathrm{C}$. A recent report by Nikolopoulos et al. [15] indicates that molten silicon wets $\alpha$-SiC more easily than $\beta$-SiC. The data on the wetting behavior of silicon-molybdenum alloys with carbon or silicon carbide are not available in the literature. Choh and Oki [16] reported that the alloying of aluminum by transition elements like molybdenum and niobium increases its wetting with silicon carbide due to the tendency of solutes to form carbides.

The objective of this paper is to study the effect of the carbon preform characteristics on the melt infiltration process. This is accomplished just by theoretically evaluating the effect of initial pore volume fraction of the preform on the amount of molybdenum disilicide

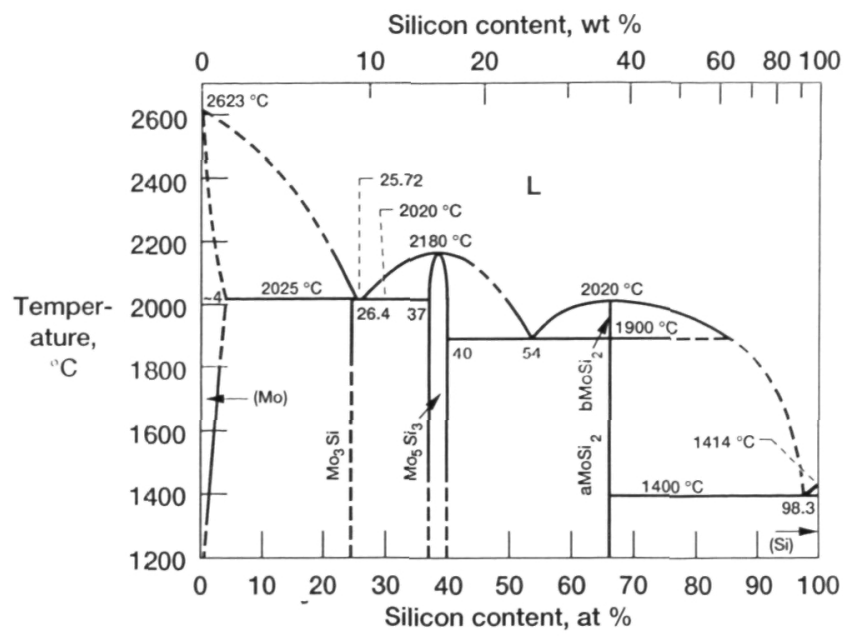

Fig. 1. Phase diagram of molybdenum-silicon system [10].

and residual silicon phases in reaction-formed silicon carbide ceramics. Next, the effect of carbon preform pore size and infiltration material (silicon and silicon-molybdenum alloys) on the exothermic reaction temperature is experimentally evaluated. Finally, the microstructures and preliminary mechanical properties (flexural strength and fracture toughness) of the reaction-formed silicon carbides are discussed.

\section{Experimental details}

The porous carbon preforms used in this study were made from a mixture of furfuryl alcohol resin, diethylene and triethylene glycol, and $p$-toluene sulphonic acid. This mixture is polymerized to form a porous solid polymer which is heated up to $700{ }^{\circ} \mathrm{C}$ to yield a porous carbon preform. By varying the ratios of the constituents in the polymer system, carbon preforms with a wide variation of pore volumes, pore sizes, and morphologies have been obtained. These porous carbon preforms have been infiltrated with silicon-1.7 and 3.2 at.\% molybdenum alloys at temperatures of $1425-1500{ }^{\circ} \mathrm{C}$ for different periods of time. The selection of processing time and temperature is critical to avoid cracking of the specimens due to thermal expansion mismatch and volume change. After infiltration, samples were cross-sectioned and polished for metallographic studies. Powder X-ray diffraction analysis was used for the identification of different phases.

Differential thermal analysis (DTA) studies have been carried out in a Netzsch STA differential thermal analyzer system using a heating rate of $10{ }^{\circ} \mathrm{C} \mathrm{min}{ }^{-1}$ in a flowing helium atmosphere. Infiltration materials (silicon-molybdenum alloys) were placed at the top of the porous carbon preform discs. Porous carbon pre- 
forms with two pore sizes, i.e. 0.2 and $1.8-2 \mu \mathrm{m}$, were used for DTA studies.

Four-point flexural strength testing of reactionformed silicon carbide materials was performed with MIL-STD-1942 (MR) configuration B with $20 \mathrm{~mm}$ inner span and $40 \mathrm{~mm}$ outer span. The flexure specimens were $50 \times 5 \times 4 \mathrm{~mm}$ in length, width, and height respectively. A loading rate of $0.5 \mathrm{~mm} \mathrm{~min}^{-1}$ was employed in the flexural test. Fracture toughness was measured using the single-edge-precracked-beam (SEPB) method. Fracture surfaces of tested specimens were examined by optical and scanning electron microscopy.

\section{Results and discussion}

Generally, the reactive infiltration of molten silicon or silicon alloys into porous carbon preforms with optimum properties leads to complete conversion of carbon to silicon carbide under suitable experimental conditions. The completely converted materials contain silicon carbide as the primary phase and resid- ual silicon or silicon and refractory disilicide as the secondary phases. However, under certain experimental conditions, it is quite possible to have residual free carbon and a small amount of porosity in the final material. Inadequate properties of the carbon preforms (non optimal pore volume, shape and/or size) lead to undesirable infiltration results. Some commonly observed undesirable features as shown in Fig. 2 are choking-off, unreacted carbon, specimen cracking, and silicon vein and lake formation. These undesirable attributes can be controlled by the careful selection of the morphology and porosity of the carbon preform and the kinetics of the reactive infiltration. In the following sections, theoretical modeling and experimental results to deal with some of these issues will be discussed in detail.

\subsection{Theoretical pore volumes}

Theoretical details of the pore volume calculations for the infiltration of silicon and silicon-refractory metal $(\mathrm{Mo}, \mathrm{Nb})$ alloy into polymer and slurry derived preforms have been presented elsewhere [17-18].
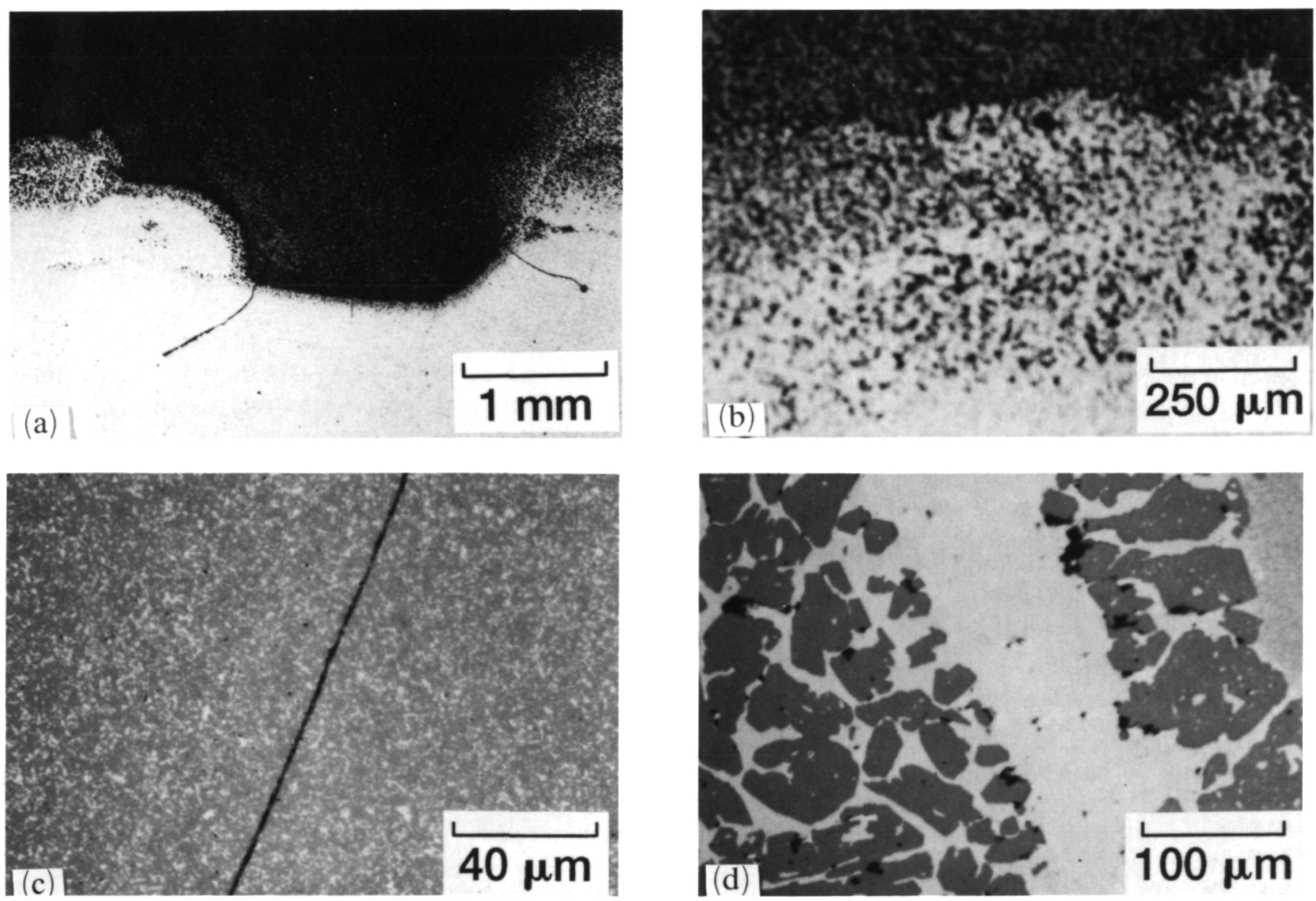

Fig. 2. Undesirable results of melt infiltration showing: (a) choking-off; (b) unreacted carbon; (c) cracking and silicon vein; and (d) silicon lake formation. 
Experimental results have shown that the external dimensions of a porous carbon preform do not change during the reactive melt infiltration process. As the reactive infiltration proceeds, the pores or channels through which the infiltrants travel become narrower, which reduces the flow of infiltrant to the reaction front. For initial pore volume fractions less than a critical value, the channels will be closed and the flow will stop. This phenomena is called choking-off of the infiltration.

The computational results obtained for the silicon$1.7 \%$ molybdenum alloy infiltration are given in Fig. 3. This plot shows the relationship between the initial pore volume fraction of the carbon preform and the volume fractions of reaction products, i.e. silicon carbide $\left(V_{\mathrm{SiC}}\right)$, molybdenum disilicide $\left(V_{\mathrm{MoSi}_{2}}\right)$, and free silicon $\left(V_{\mathrm{Si}}\right)$ in the reaction-formed silicon carbide material. According to these results, for initial pore volume fractions less than 0.379 , the porous carbon preform will not be completely converted due to choking-off of the infiltration. The infiltration passages become narrow and are ultimately closed as a result of the specific volume change during conversion from carbon to silicon carbide. The preforms with an initial pore volume fraction of 0.379 will yield silicon carbide and molybdenum disilicide. Preforms with greater than 0.379 pore volume fraction give final materials which have silicon carbide with molybdenum disilicide and silicon phases. The dependence of the choking-off pore volume fraction on the concentration of molybdenum in the silicon-molybdenum alloy is given in Fig. 4.

The preforms used in this work were made by the pyrolysis of a high-char yield polymer, which resulted in a porous glassy carbon preform. The density of glassy carbon is $1.5 \mathrm{~g} \mathrm{~cm}^{-3}$. However, the density values for the other carbon types, e.g. graphite, can also be used in the computations as shown in Fig. 4.

The capillary flow and reaction of silicon-molybdenum alloy in pore channels and around carbon particles results in the formation of silicon carbide and the precipitation of molybdenum disilicide from the alloy melt. The reaction of carbon with molten siliconmolybdenum alloy is as follows:

$$
\begin{aligned}
\mathrm{C}(\mathrm{s})+\frac{(1+2 x+y) \mathrm{Si}(1)+x \mathrm{Mo}(1)}{\mathrm{Si}-\mathrm{Mo} \text { alloy }} \\
=\mathrm{SiC}(\mathrm{s})+x \mathrm{MoSi}_{2}(\mathrm{~s})+y \mathrm{Si}(\mathrm{s})
\end{aligned}
$$

The amount of molybdenum disilicide in the final material depends on the concentration of molybdenum $(x)$ in the starting Si-Mo alloy. The liquid siliconcarbon reaction is exothermic with an adiabatic temperature $\left(T_{\mathrm{ad}}\right)$ of $1775 \mathrm{~K}$. The adiabatic temperature calculations for the reaction of silicon and molyb-

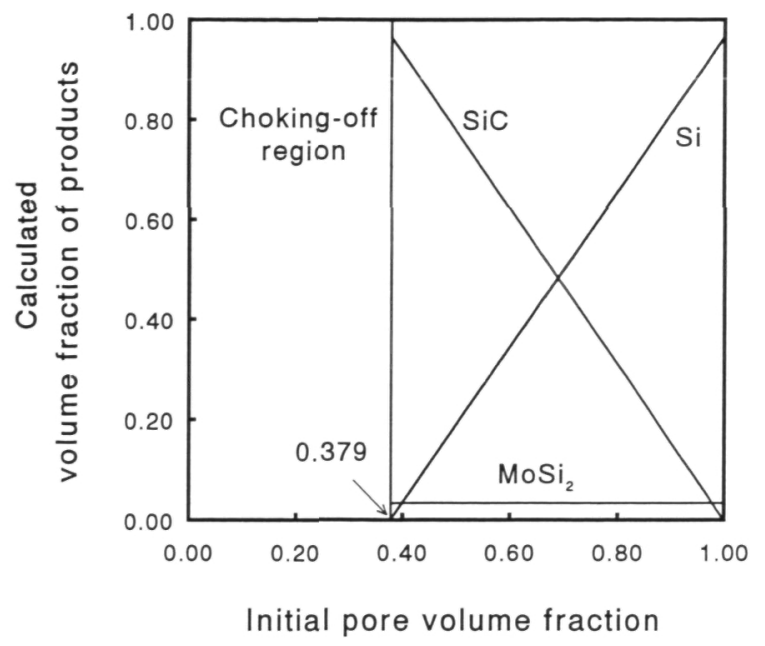

Fig. 3. Effect of initial pore volume fraction of carbon preform on the volume fractions of reaction products.

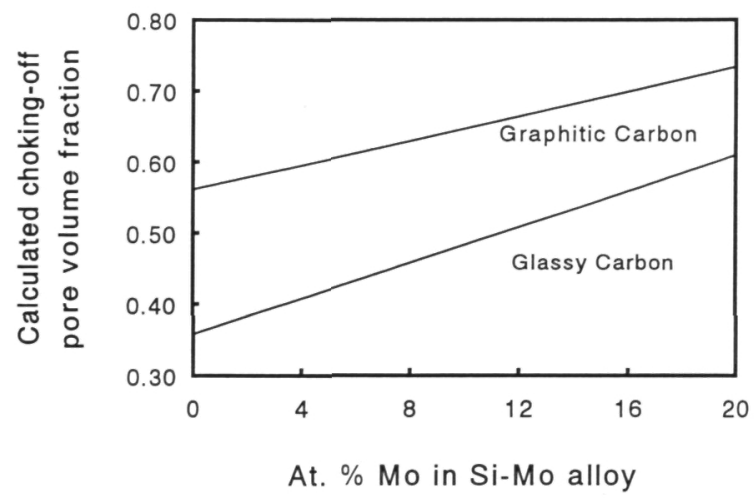

Fig. 4. Dependence of choking-off pore volume fraction on the concentration of Mo in the $\mathrm{Si}-\mathrm{Mo}$ alloys.

denum with carbon indicate that there is a reduction in the adiabatic temperature with increase in the amount of molybdenum in the reactant [19].

\subsection{DTA studies}

Our experimental results [4-5] and literature data [20-21] confirm that the reaction of porous carbon with molten silicon and silicon alloys is exothermic and the reaction time is very short. The degree of exothermicity of this reaction depends on a number of parameters including the surface area of the reactants (pore size and the pore volume of porous carbon) and wetting behavior. DTA curves for the reaction of a small pore size $(\sim 0.2 \mu \mathrm{m})$ carbon will silicon and silicon-3.2 at.\% molybdenum alloy are given in Fig. 5 (a) and (b). It is clearly evident from this figure that the exothermic temperature in the case of alloy infiltration 


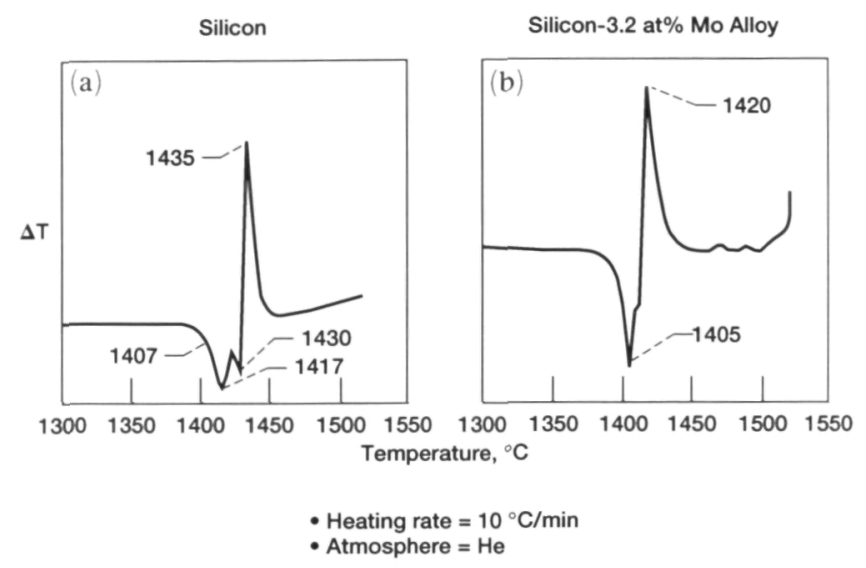

Fig. 5. DTA curves for the reaction of small pore size carbon preform with (a) $\mathrm{Si}$ and (b) $\mathrm{Si}-3.2$ at.\% Mo alloy. Heating rate $=10{ }^{\circ} \mathrm{C} \min ^{-1}$, atmosphere $=\mathrm{He}$.

is lower than that with pure silicon. In silicon experiments, there is another endotherm at $1430{ }^{\circ} \mathrm{C}$ in addition to one due to melting of silicon. However, there is no similar endotherm in the silicon-3.2 at.\% molybdenum alloy system. This phenomenon may be explained on the basis of thermodynamics of the liquid silicon and carbon reaction and the nucleation and growth of the second phase. Owing to the exothermic nature of the reaction, under certain conditions, the dissolution of carbon takes place in molten silicon solution and silicon carbide precipitates [5].

The exothermic reaction temperatures observed in the DTA studies for the reaction of medium pore size $(\sim 1.8-2 \mu \mathrm{m})$ carbon preforms are given in Fig. 6 (a) and (b). In Fig. 6(a), there is an initial incubation period in the reaction (observed via DTA) before the exotherm starts. The cause of this phenomenon has been speculated by various researchers for the case of carbon fiber-liquid silicon reaction $[8,20,21]$. The exotherm temperature in the case of silicon-3.2 at.\% molybdenum alloy is again lower than for the pure silicon.

In order to explain the reaction mechanism of the infiltrant(s)-carbon system, the liquid silicon-carbon system is chosen for discussion (that is, without any alloying of the silicon melt). For this system, there is a higher exothermic reaction temperature of liquid silicon with medium pore size carbon in comparison with the exothermic reaction temperature of small pore size carbon preforms. One reason for this may be the fact that in the case of small pore size carbon preforms, the reaction and infiltration front move almost at the same time. But in the case of the medium pore size preform, the infiltration front moves faster than the

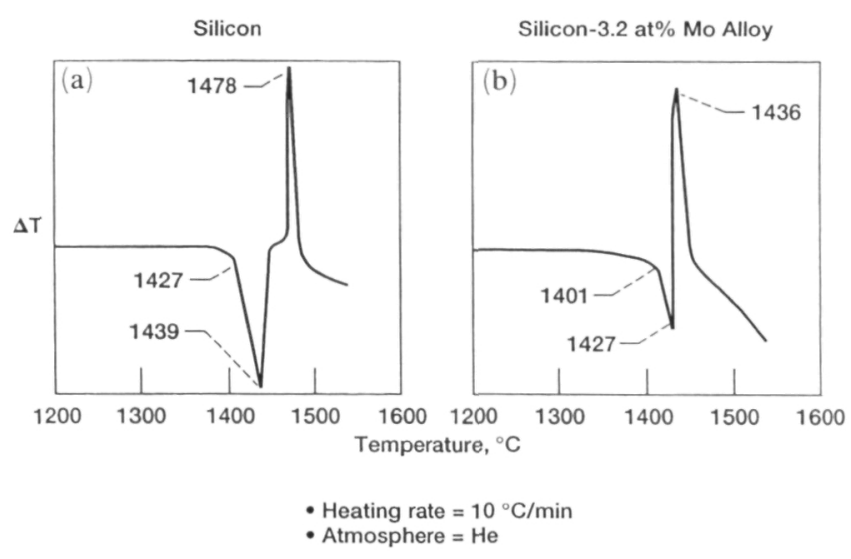

Fig. 6. DTA curves for the reaction of medium pore size carbon preform with (a) $\mathrm{Si}$, and (b) $\mathrm{Si}-3.2$ at.\% Mo alloy. Heating rate $=10^{\circ} \mathrm{C} \min ^{-1} ;$ atmosphere $=\mathrm{He}$.

reaction front. Liquid silicon infiltrates throughout the preform and there is no control over the reaction. This leads to the higher exothermic reaction temperatures.

In the reactive infiltration process reported here, the controlling factors are the wetting and infiltration of the porous carbon preform by molten silicon. The formation of primary silicon carbide is a result of the heterogeneous reaction of carbon with silicon melt and subsequent crystallization of $\mathrm{SiC}(\beta)$. The reactions occurring at various interfaces are as follows:

$$
\begin{aligned}
& \mathrm{Si}(1)+\mathrm{C}(\mathrm{s})=\mathrm{SiC}(\mathrm{s}) \\
& \mathrm{Si}(1)+\mathrm{C}(\mathrm{s})=\mathrm{Si} / \mathrm{C}(\mathrm{sol}) \\
& \mathrm{Si} / \mathrm{C}(\mathrm{sol})=\mathrm{SiC}(\mathrm{s})
\end{aligned}
$$

Recent studies by various authors [5,8,20,21,23] confirm that the primary silicon carbide dissolves in the molten silicon and then reprecipitates as secondary silicon carbide. Owing to the low coefficients of diffusion of carbon and silicon in silicon carbide, the growth of silicon carbide by solid state reaction at the carbon-liquid silicon interface should be insignificant. In addition, the diffusion coefficients of carbon in silicon carbide are two orders of magnitude higher than that of silicon in silicon carbide [22].

On the other hand, for the alloy-carbon system, two main factors influence the alloy infiltration. First, due to molybdenum alloying of silicon, the wetting behavior with carbon improves due to its tendency to form molybdenum carbide $\left(\mathrm{Mo}_{2} \mathrm{C}\right)$ [16]. Second, the molybdenum disilicide precipitates may also act as a heat sink leading to a reduction in the exotherm for the reaction of silicon-3.2 at.\% molybdenum alloy with carbon. 


\subsection{Melt infiltration studies}

A scanning electron micrograph of the fracture surface of a medium pore size carbon preform is given in Fig. 7. This micrograph shows uniform struts and pores indicating the ability of this process to produce carbon preforms with homogeneous microstructures. Fig. 8 is the cross-section of a silicon-3.2 at.\% molybdenum alloy infiltrated preform (medium pore size), showing the complete conversion of carbon by the reactive melt infiltration of the alloy, and uniform distribution of molybdenum disilicide, and silicon phase throughout the silicon carbide matrix. The X-ray diffraction pattern of this material indicates the presence of silicon carbide, molybdenum disilicide and residual silicon phase. There is no evidence of other

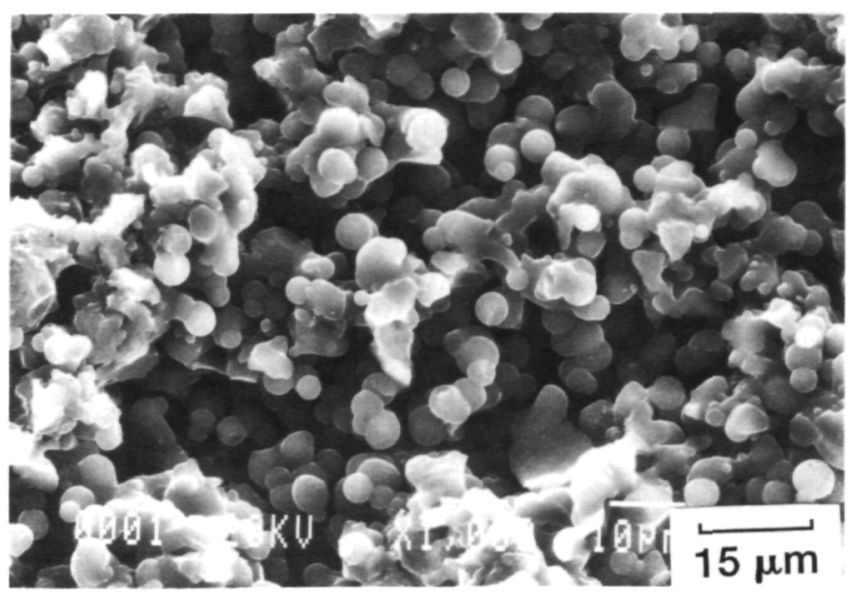

Fig. 7. Micrograph of the fracture surface of a porous carbon preform.

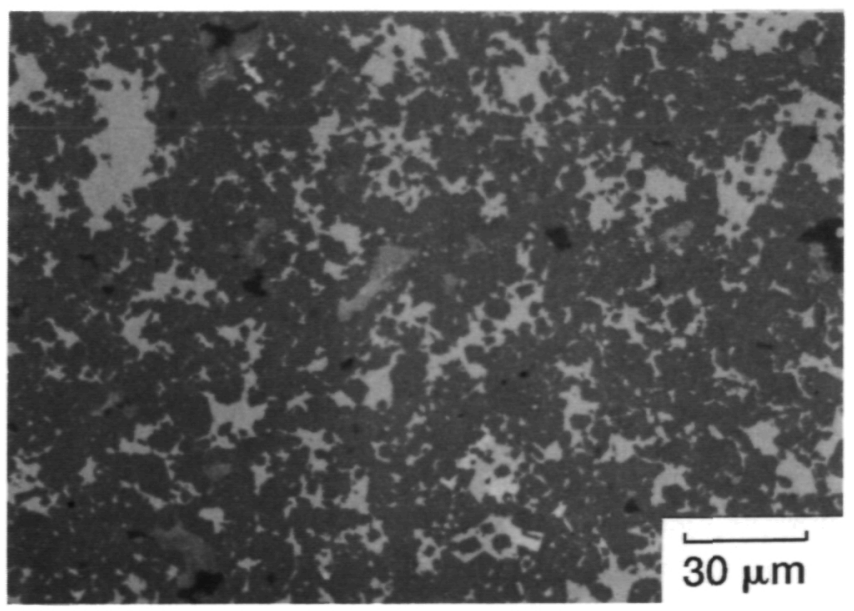

Fig. 8. Polished cross-section of $\mathrm{Si}-3.2$ at.\% Mo alloy infiltrated material. binary or ternary phases of molybdenum-carbon or molybdenum-silicon-carbon. The uniformity of the microstructure indicates that the alloying of the melt is quite useful for introducing uniformly distributed second phases in reaction-formed silicon carbide materials.

\subsection{Mechanical properties}

Room-temperature flexural properties of the reaction-formed silicon carbide materials described above were determined using the four-point bend technique. The average value of flexural strength for five samples was $288 \pm 15 \mathrm{MPa}$. The room-temperature fracture toughness of this material, determined using the singleedge-precracked-beam (SEPB) method, was $3.3 \pm 0.2$ $\mathrm{MPam}^{1 / 2}$. This value is the average of three specimens. There is a slight improvement in the toughness over commercial reaction-bonded silicon carbide as well as our silicon containing reaction-formed silicon carbide materials $\left(2.5 \pm 0.2 \mathrm{MPa} \mathrm{m}^{1 / 2}\right)[24]$.

The fracture surfaces of these specimens were examined under the scanning electron microscope. The mirrors were broad and poorly defined. However, some fracture surfaces exhibited optically reflective mirror-like regions which contained directional fracture lines pointing to the fracture origin at the tensile surface. The strength-limiting flaws in this material included unreacted carbon, pores, and surface damage caused by machining. The failure mode was typically brittle and cracks either originated at the machining flaws at the surface or at unreacted carbon inside the specimen. Fig. 9 shows a typical brittle failure of material with $320 \mathrm{MPa}$ strength. Fig. 10 shows the fracture surface of a lower-strength bar where the failure origin is unreacted carbon in the material.

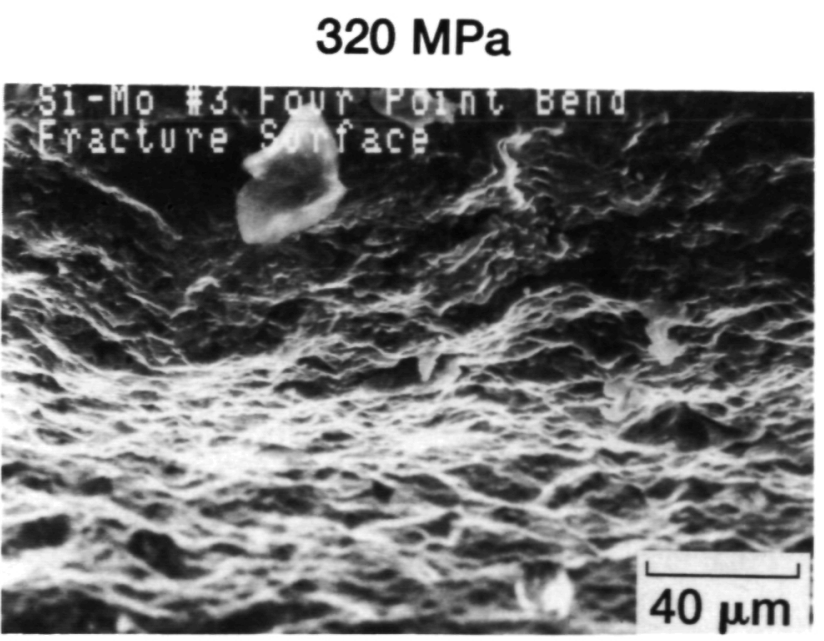

Fig. 9. Fractograph showing a typical brittle fracture. 
$220 \mathrm{MPa}$

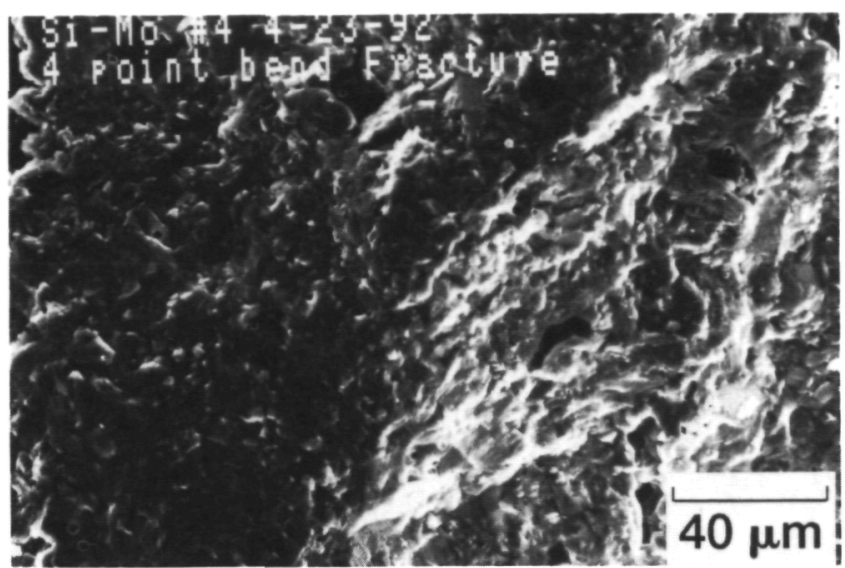

Fig. 10. Unreacted carbon in the sample as the origin of flaw.

molybdenum alloy requires higher initial pore volume fraction in the preform to prevent the choking-off. The mechanism of silicon-carbon reaction is a combination of interface-limited and solution reprecipitation type and depends on the pore and particle size of the carbon. The silicon-molybdenum alloy infiltrants result in in-situ formed molybdenum disilicide phase which is advantageous in improving the fracture toughness of reaction-formed silicon carbide materials.

\section{Acknowledgments}

This work was done while one of the authors (M.S.) held a National Research Council-NASA Senior Research Associateship at Lewis Research Center. We would like to thank Mr. R.F. Dacek for specimen preparation and Mr. R. Pawlik for mechanical testing.

\section{References}

\section{Summary of results}

Using the combination of modeling, melt infiltration, and DTA results, it has been concluded that the initial pore volume and pore size of the carbon preform are very critical parameters in the melt infiltration process. Modeling results indicate that carbon preforms with initial pore volume fractions of 0.379 and higher can be infiltrated with silicon-1.7 at.\% molybdenum alloy. The initial pore volume fraction needed to prevent the choking-off increases with the increasing content of molybdenum in the alloy. DTA results indicate that the lower reaction exotherms can be obtained using silicon-molybdenum alloy infiltration instead of pure silicon and by using small pore size $(-0.2 \mu \mathrm{m})$ carbon preforms. The reaction mechanism of carbon with silicon is a combination of interface-limited and solution reprecipitation type, depending on the pore and particle size of the carbon.

Preliminary evaluation of strength data suggest that the strength of these materials appears to be limited by machining flaws and the presence of processing-related flaws such as unreacted carbon.

\section{Conclusions}

The findings of this work suggest that use of silicon-molybdenum alloys instead of pure silicon as the infiltrant has beneficial effects in terms of reduced reaction exotherm temperature during the processing of reaction-formed silicon carbide ceramics. The increasing amount of molybdenum in the silicon-

1] E. Fitzer and R. Gadow, Am. Ceram. Soc. Bull., 65 (1986) 325-35.

[2] M.E. Washburn and W.S. Coblenz, Ceram. Bull., 67(1988) 356-63.

[3] E.E. Hucke, AMMRC Report TR-83-5, Defense Technical Info. Center, Arlington, VA, January 1983.

[4] D.R. Behrendt, M. Singh and R.F. Dacek, Proc. 16th Conf. on Metal, Carbon and Ceramic Matrix Composites, NASA CP-3175, Cocoa Beach, FL, 1992, pp. 695-705.

[5] M. Singh and D.R. Behrendt, NASA-TM-105860, Lewis Research Center, Cleveland, OH, 1992.

[6] R.P. Messner and Y.-M. Chiang, J. Am. Ceram. Soc., 73 (1990) 1193-1200.

[7] C.B. Lim, T. Yano and T. Iseki, J. Mater. Sci., 24 (1989) 4144-151.

[8] Y.-M. Chiang, R.P. Messner, C. Terwilliger and D.R. Behrendt, Mater. Sci. Eng., A144 (1991) 63-74.

[9] A.K. Vasudevan and J.J. Petrovic, Mater. Sci. Eng., A155 (1992) 1-17.

[10] Binary Alloy Phase Diagrams,, American Society for Metals, Metals Park, OH, 1990.

[11] H. Nowtony, E. Parthe, R. Kieffer and F. Benesovsky, Monatsch. Chem., 85 (1954) 255.

[12] Ju. V. Naidich, The wettability of solids by liquid metals, in D.A. Cadenhead and J.F. Danielli, (eds.), Progress in Surface and Membrane Science, New York, 1981, pp. 353-484.

[13] J.G. Li and H. Hausner, J. Mater. Sci. Lett., 10 (1991) $1275-76$.

[14] G.G. Gnesin and A.I. Raichenko, Poroshk. Metall., 5 (1973) 35-43.

[15] P. Nikolopoulos, S. Agathopoulos, G.N. Angelopoulos, An Naoumidis and H. Grubmeier, J. Mater. Sci., 27 (1992) 139-45.

[16] T. Choh and T. Oki, Mater. Sci. Technol., 3(1987) 378-85.

[17] D.R. Behrendt and M. Singh, J. Mater. Synth. Proc., 2 (1994) 133-39.

[18] D.R. Behrendt and M. Singh, NASA-TM-106414, Lewis Research Center, Cleveland, OH, 1993. 
[19] M. Singh, unpublished work.

[20] R. Pampuch, E. Walasek and J. Bialoskorski, Ceram. Int., 12(1986) 99-106.

[21] R. Pampuch, J. Bialoskorski and E. Walasek, Ceram. Int., 13 (1987) 63-8.

[22] J.D. Hong and R.F. Davis, J. Am. Ceram. Soc., $63(1980)$
$546-52$.

[23] J.N. Ness and T.F. Page, J. Mater. Sci., 21 (1986) 1377-97.

[24] M. Singh, R. Pawlik, J.A. Salem and D.R. Behrendt, in N.P. Bansal (ed.), Ceram. Trans., Vol. 38, Advances in Ceramic Matrix Composites, American Ceramic Society, Westerville, OH, 1993 pp. 349-60. 


\section{Instructions for Authors}

\section{SUBMISSION OF PAPERS}

Manuscripts for the main part of the journal should be submitted to the Editor-in-Chief, Professor H. Herman, or for authors in Japan to Professor M. Koiwa:

\section{Professor Herbert Herman}

Department of Materials Science and Engineering

State University of New York at Stony Brook

Long Island, NY 11794-2275, USA

Fax: +1 (516) 6328052

Professor Masahiro Koiwa

Department of Metal Science and Technology

Faculty of Engineering

Kyoto University

Yoshida-Honmachi, Sakyo-ku

Kyoto 606-01, Japan

Fax: +81 (75) 7517844

Manuscripts for the Letters Section should be submitted as follows:

For authors in Europe

Professor G. Kostorz

ETH Zurich

Institut für Angewandte Physik

$\mathrm{CH}-8093$ Zurich, Switzerland

Fax: + 41 (1633) 1105

For authors in Japan

Professor Masahiro Koiwa

Japan

For authors in North and South America and the rest of the world

Professor Herbert Herman

USA

\section{Manuscripts}

Three copies should be submitted to the Editor, in double-spaced typing on pages of A4 size and with wide margins (Letters should not exceed 2000 words and a maximum of 5 figures). All tables and illustrations should bear a title or legend.
An abstract should accompany reviews, original papers and Letters. It should present (preferably in 100-150 words; 50 words or less for Letters) a brief and factual account of the contents and conclusions of the paper, and an indication of the relevance of new material.

References should be indicated by numerals in square brackets, introduced consecutively and appropriately in the text.

References must be listed on separate sheet(s) at the end of the paper. Every reference appearing in the text should be quoted in the reference list, and vice versa. When reference is made to a publication written by more than two authors it is preferable to give only the first author's name in the text followed by "et al.". However, in the list of references the names and initials of al authors must be given.

Three sets of figures should be submitted. One set of line drawings should be in a form suitable for reproduction, drawn in Indian ink on drawing or tracing paper (letter height, 3-5 $\mathrm{mm}$ ). Alternatively, such illustrations may be supplied as high contrast, black-and-white glossy prints. Duplicate original micrographs should be provided wherever possible to facilitate the refereeing process. Magnifications should be indicated by a ruled scale bar on the micrograph. Captions to illustrations should be typed in sequence on a separate page.

All abbreviated terms must be defined when first used (both in the abstract and in the text) and authors must express all quantities in SI units, with other units in parentheses if desired.

Authors in Japan please note that information about how to have the English of your paper checked, corrected and improved (before submission) is available from: Elsevier Science (Japan), 20-12 Yushima 3-chome, Bunkyo-ku, Tokyo 113; Tel: (03) 38333821; Fax: (03) 3836-3064.

\section{Further information}

All questions arising after the acceptance of manuscripts, especially those relating to proofs, should be directed to: Elsevier Editorial Services, Mayfield House, 256 Banbury Road, Oxford OX2 7DH, UK (tel. +44 1865 314900; fax. +44 1865 314990).

No part of this publication may be reproduced, stored in a retrieval system or transmitted in any form or by any means, electronic, mechanical, photocopying, recording or otherwise, without the prior written permission of the publisher, Elsevier Science SA, P.O. Box 564, 1001 Lausanne, Switzerland.

Submission of an article for publication implies the transfer of the copyright from the author(s) to the publisher and entails the author(s) irrevocable and exclusive authorization of the publisher to collect any sums or consideration for copying or reproduction payable by third parties.

Upon acceptance of an article by the journal, the author(s) will be asked to transfer copyright of the article to the publisher. This transfer will ensure the widest possible dissemination of information.

\section{For Material Subject to US Copyright Law}

Special regulations for readers in the USA

This journal has been registered with the Copyright Clearance Center, Inc., 222 Rosewood Drive, Danvers, MA 09123, USA. Consent is given for copying of articles for personal use, or for the personal use of specific clients. This consent is given on the condition that the copier pays through the Center the per-copy fee stated in the code on the first page of each article for copying beyond that permitted by Sections 107 or 108 of the US Copyright Law. If no code appears in an article, the author has not given broad consent to copy and permission to copy must be obtained directly from the author. All articles published prior to 1982 may be copied for a per-copy fee of US $\$ 2.50$, also payable through the Center. This consent does not extend to other kinds of copying, such as for general distribution, resale, advertising and promotion purposes or for creating new collective works. Special written permission must be obtained from the publisher for such copying.

No responsibility is assumed by the Publisher for any injury and/or damage to persons or property as a matter of products liability, negligence or otherwise, or from any use or operation of any methods, products, instructions or ideas contained in the material herein. 